\title{
On the Euler characteristic of definable groups
}

\author{
Mário J. Edmundo*1 \\ ${ }^{1}$ Universidade Aberta and CMAF - UL, Av. Prof. Gama Pinto 2, 1649-003 Lisboa, Portugal
}

\begin{abstract}
Key words O-minimal structures, definable groups. MSC (2000) 03C64; 20E99

We show that in an arbitrary o-minimal structure the following are equivalent: (i) conjugates of a definable subgroup of a definably connected, definably compact definable group cover the group if the o-minimal Euler characteristic of the quotient is non zero; (ii) every infinite, definably connected, definably compact definable group has a non trivial torsion point.
\end{abstract}

Copyright line will be provided by the publisher

\section{Introduction}

We work over an arbitrary o-minimal structure $\mathcal{N}$ and definable means $\mathcal{N}$-definable (possibly with parameters). We will assume that the reader is familiar with the basic theory of o-minimality ([7]) and of definable groups ([8], [19], [20], [21], [22], [23] and [24]).

In o-minimal structures there is an Euler-Grothendieck characteristic (in the sense of [16]) for the category of definable sets, denoted $E(-)$, which is useful for analyzing definable groups as the work by Strzebonski ([25]) shows. In this paper, following the line of Strzebonski's work combined with results from [10], we prove the following:

Theorem 1.1 In an arbitrary o-minimal structure the following are equivalent:

1. If $H$ is a definable subgroup of a definably connected, definably compact, definable group $G$ such that $E(G / H) \neq 0$, then there exists a definably connected, definably compact definable abelian subgroup $C$ of $G$ such that $C \subseteq H, \operatorname{dim} C$ is the maximal dimension of abelian definable subgroups of $G$ and $G=$ $\cup\left\{g C g^{-1}: g \in G\right\}$. In particular, $G=\cup\left\{g H g^{-1}: g \in G\right\}$.

2. Every infinite, definably connected, definably compact definable group G has a non trivial torsion point.

\section{The main result and other observations}

Here we prove the main result of the paper and make some other observations that follow from it. Clearly in Theorem 1.1 condition (1) implies condition (2). Indeed, suppose that $G$ has no non trivial torsion points. Since $E(G)=0$ implies that $G$ has a $k$-torsion point for every $k \in \mathbf{N}$ ([25] Lemma 2.5$), E(G) \neq 0$. Now take $H=1$ to reach a contradiction.

To verify that in Theorem 1.1 condition (2) implies condition (1) we will require the following perhaps well known observation:

Lemma 2.1 Let $G$ be a definable connected, definably compact definable group. If $G$ has a non trivial torsion point then $E(G)=0$ and $G$ has non trivial $k$-torsion points for every $k \in \mathbf{N}$.

Proof. If $G$ has a non trivial torsion point, then it has a $p$-torsion point $x$ for some prime $p$. By divisibility of $G$ ([10]) for every $l \in \mathbf{N}$ there is $z_{l} \in G$ such that $\left(z_{l}\right)^{p^{l}}=x$. Then $z_{l}$ is a non trivial $p^{l+1}$-torsion point of $G$. Let $C_{l}$ be the subgroup of $G$ of order $p^{l+1}$ generated by $z_{l}$. Then there is a definable bijection between $C_{l} \times G / C_{l}$ and $G$, and consequently $E(G)=E\left(G / C_{l}\right) E\left(C_{l}\right)=E\left(G / C_{l}\right) p^{l+1}$. So $p^{l+1}$ divides $E(G)$ and we must have

\footnotetext{
* Corresponding author: e-mail: edmundo@cii.fc.ul.pt
} 
$E(G)=0$ since $l$ is arbitrary. Now by [25] Lemma 2.5, $E(G)=0$ implies that $G$ has a $k$-torsion point for every $k \in \mathbf{N}$.

So assume condition (2) of Theorem 1.1. Then by the Lemma 2.1 every infinite, definably connected, definably compact definable group has o-minimal Euler characteristic zero. Let $G$ be a definably connected, definably compact definable group and suppose that $H$ is a definable subgroup of $G$ such that $E(G / H) \neq 0$.

Consider the definably connected component $H^{0}$ of $H$. By [25] Theorem 2.14, there is a definable bijection from $G / H \times H / H^{0}$ to $G / H^{0}$. So $E(G / H) E\left(H / H^{0}\right)=E\left(G / H^{0}\right)$. Since $E\left(H / H^{0}\right)=\left(H: H^{0}\right) \neq 0$, it follows that $E\left(G / H^{0}\right) \neq 0$. As $H^{0}$ is definably connected and definably compact, by [10] Proposition 1.2, there exists a definably connected, definably compact definable abelian subgroup $C$ of $H^{0}$ which is unique up to conjugation such that $\operatorname{dim} C$ is the maximal dimension of abelian definable subgroups of $H^{0}$ and $H^{0}=$ $\cup\left\{h C h^{-1}: h \in H^{0}\right\}$. By [25] Theorem 2.14, there is a definable bijection from $G / H^{0} \times H^{0} / C$ to $G / C$. So $E\left(G / H^{0}\right) E\left(H^{0} / C\right)=E(G / C)$.

We claim that $C$ is a definable 0 -subgroup of $H^{0}$ and also of $G$ in the sense of [25], i.e., for every proper definable subgroup $L$ of $C$ we have $E(C / L)=0$. Indeed, if $L$ is a proper definable subgroup of $C$, then $C / L$ is an infinite definably connected, definably compact definable group ([8] Lemmas 3.14 and 3.15), and the assumption implies that $E(C / L)=0$ as required. By maximality of $\operatorname{dim} C$ we have $E\left(H^{0} / C\right) \neq 0$. In fact, the condition $E\left(H^{0} / C\right)=0$ implies there is a definable 0-subgroup $K$ of $H^{0}$ with $C<K<N_{H^{0}}(C)$ and $C \neq K\left([25]\right.$ Theorem 2.14). Now from the fact that $E\left(G / H^{0}\right) E\left(H^{0} / C\right)=E(G / C)$ and $E\left(G / H^{0}\right) \neq 0$ we conclude that $E(G / C) \neq 0$. Thus $C$ is a 0 -Sylow definable subgroup in the sense of [25], i.e., it a maximal definable 0-subgroup of $G$ (this follows at once from the definitions and [25] Theorem 2.14). In particular, $\operatorname{dim} C$ is the maximal dimension of abelian definable subgroups of $G$. Now by [25] Theorem 2.21 we have also that $G=\cup\left\{g C g^{-1}: g \in G\right\}$.

Corollary 2.2 Suppose that $\mathcal{N}$ is an o-minimal expansion of an ordered group. If $H$ is a definable subgroup of a definably connected, definably compact definable group $G$ such that $E(G / H) \neq 0$, then $G=\cup\left\{g H g^{-1}\right.$ : $g \in G\}$.

Proof. By Theorem 1.1 it is enough to verify (2). In the abelian case this has been verified in [15] (linear case), [18] (semi-bounded non linear case) and in [12] (field case). For the general case one can use the fact that $G / Z(G)^{0}$ is definably semi-simple ([20] or [8]), transfer and the description of such groups given in [21], [22] and [23].

We end the paper with the following observations:

- Corollary 2.2 was previously proved in o-minimal expansions of fields in [1] using the o-minimal Lefschetz fixed point theorem ([2], [11] and [14]).

- In o-minimal expansions of fields, there is another short proof of the existence of non trivial torsion points on infinite, definably connected, definably compact definable abelian groups besides the ones that appear in [2] and in [12]. This alternative proof avoids also the o-minimal Lefschetz fixed point theorem. Indeed, using the o-minimal singular homology ([13] and [26]) one can define the Lefschetz number of the multiplication by $k$ map on such abelian definable group $G$. As in the classical book by Brown ([4], Chapter III, Section F) one can show that this number is $(1-k)^{r}$ for a suitable $r$ (which is positive because $G$ is orientable). Hence the Lefschetz number of the identity on $G$, which is also the o-minimal Euler characteristic of $G$ ([2], p. 788), is zero. Hence by [25] Lemma $2.5, G$ has a $k$-torsion point for every $k \in \mathbf{N}$. This argument was first pointed out by the author in the unpublished manuscript [9].

- Connected, compact Lie groups are semi-algebraic groups: any compact Lie group $G$ is isomorphic to a compact subgroup $K$ of $G L(n, \mathbf{R})$ for some $n$ ([5], Chapter 3, Theorem 4.1, p.136) and any such $K$ is a (real)algebraic subgroup of $G L(n, \mathbf{R})$ ([6], Proposition 2, p. 230). Thus the results of this paper together with those from [10] generalize classical results about compact Lie groups (see for example [17], Chapter V, Section 3). 
Acknowledgements The author was supported by the FCT (Fundação para a Ciência e Tecnologia) program POCTI (Portugal/FEDER-EU) and FCT (Fundação para a Ciência e Tecnologia) project PTDC/MAT/101740/2008.

\section{References}

[1] A. Berarducci Zero-groups and maximal tori in: Logic Colloquium 2004. Editors: A. Andretta, K. Kearnes, D. Zambella, ASL Lecture Notes in Logic 29 (2006) 33-45.

[2] A. Berarducci and M. Otero Transfer methods for o-minimal topology J. Symb. Logic 68 (3) (2003) 785-794.

[3] A. Berarducci, M. Edmundo and M. Otero Corrigendum to "Transfer methods for o-minimal topology" J. Symb. Logic 72 (3) (2007) 1079-1080.

[4] R. Brown The Lefschetz fixed point theorem Scott, Foresman and Company 1971.

[5] T. Bröcker and T. tom Dieck Representations of compact Lie groups vol. 98 Springer-Verlag 1985.

[6] C. Chevalley Théorie des groupes de Lie. Tome III. Théorèmes généraux sur les algèbres de Lie Hermann \& Cie 1955.

[7] L. van den Dries Tame topology and o-minimal structures Cambridge University Press 1998.

[8] M. Edmundo Solvable groups definable in o-minimal structures J. Pure Appl. Algebra 185 (1-3) (2003) 103-145.

[9] M. Edmundo O-minimal cohomology and definably compact definable groups Manuscript Dec. 16, 2001. Revised May 6, 2003, 85pp.

[10] M. Edmundo A remark on divisibility of definable groups Math. Logic Quart. 51 (6) (2005) 639-641.

[11] M. Edmundo A fixed point theorem in o-minimal structures Ann. Inst. Fourier Grenoble 57 (5) (2007) 1441-1450.

[12] M. Edmundo and M. Otero Definably compact abelian groups J. Math. Logic 4 (2) (2004) 163-180.

[13] M. Edmundo and A. Woerheide Comparison theorems for o-minimal singular (co)homology Trans. Amer. Math. Soc. 360 (9) (2008) 4889-4912.

[14] M. Edmundo and A. Woerheide The Lefschetz coincidence theorem in o-minimal expansions of fields Topology Appl. 156 (15) (2009) 2470-2484.

[15] P. Eleftheriou and S. Starchenko Groups definable in ordered vector spaces over ordered division rings J. Symb. Logic 72 (2007) 1108-1140.

[16] J. Krajicek and T. Scanlon Combinatorics with definable sets: Euler characteristics and Grothendieck rings Bull. Symb. Logic 6 (2000) 311-330.

[17] M. Mimura and H. Toda Topology of Lie groups I and II Translations of Mathematical Monographs 91 AMS 1991.

[18] Y. Peterzil Returning to semi-bounded sets Preprint Oct. 2007.

[19] Y. Peterzil and C. Steinhorn Definable compacteness and definable subgroups of o-minimal groups J. London Math. Soc. 59 (2) (1999) 769-786.

[20] Y. Peterzil and S. Starchenko Definable homomorphisms of abelian groups definable in o-minimal structures Ann. Pure Appl. Logic 101 (1) (1999) 1-27.

[21] Y. Peterzil, A. Pillay and S. Starchenko Definably simple groups in o-minimal structures Trans. Amer. Math. Soc. 352 (10) (2000) 4397-4419.

[22] Y. Peterzil, A. Pillay, S. Starchenko Simple algebraic and semialgebraic groups over real closed fields Trans. Amer. Math. Soc. 352 (10) (2000) 4421-4450.

[23] Y. Peterzil, A. Pillay and S. Starchenko Linear groups definable in o-minimal structures J. Algebra 247 (2002) 1-23.

[24] A. Pillay On groups and fields definable in o-minimal structures J. Pure Appl. Algebra 53 (1988) 239-255.

[25] A. Strzebonski Euler characteristic in semialgebraic and other o-minimal groups J. Pure Appl. Algebra 96 (1994) 173-201.

[26] A. Woerheide O-minimal homology $\mathrm{PhD}$. Thesis (1996), University of Illinois at Urbana-Champaign. 